\title{
Quick Attach Docking Interface for Lunar Electric Rover
}

\author{
Jason M. Schuler ${ }^{1}$, Andrew J. Nick ${ }^{2}$, and Christopher Immer ${ }^{3}$ \\ Arctic Slope Regional Corporation (ASRC)Aerospace, Kennedy Space Center, Florida, 32899, \\ USA \\ Robert P. Mueller ${ }^{4}$ \\ National Aeronautics \& Space Administration (NASA) Kennedy Space Center, KSC, Florida, \\ 32899, USA
}

The NASA Lunar Electric Rover (LER) has been developed at Johnson Space Center as a next generation mobility platform. Based upon a twelve wheel omni-directional chassis with active suspension the LER introduces a number of novel capabilities for lunar exploration in both manned and unmanned scenarios. Besides being the primary vehicle for astronauts on the lunar surface, LER will perform tasks such as lunar regolith handling (to include dozing, grading, and excavation), equipment transport, and science operations. In an effort to support these additional tasks a team at the Kennedy Space Center has produced a universal attachment interface for LER known as the Quick Attach. The Quick Attach is a compact system that has been retro-fitted to the rear of the LER giving it the ability to dock and undock on the fly with various implements. The Quick Attach utilizes a two stage docking approach; the first is a mechanical mate which aligns and latches a passive set of hooks on an implement with an actuated cam surface on LER. The mechanical stage is tolerant to misalignment between the implement and the LER during docking and once the implement is captured a preload is applied to ensure a positive lock. The second stage is an umbilical connection which consists of a dust resistant enclosure housing a compliant mechanism that is optionally actuated to mate electrical and fluid connections for suitable implements. The Quick Attach system was designed with the largest foreseen input loads considered including excavation operations and large mass utility attachments. The Quick Attach system was demonstrated at the Desert Research And Technology Studies (D-RATS) field test in Flagstaff, AZ along with the lightweight dozer blade LANCE. The LANCE blade is the first implement to utilize the Quick Attach interface and demonstrated the tolerance, speed, and strength of the system in a lunar analog environment.

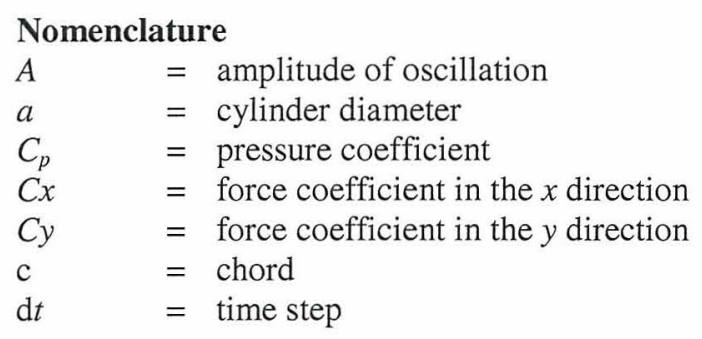

${ }^{1}$ Mechanical Engineer, Advanced Systems Division, Mail Stop ASRC-15, AIAA Member Grade for first author.

${ }^{2}$ Mechanical Engineer, Advanced Systems Division, Mail Stop ASRC-15, AIAA Member Grade for first author.

${ }^{3}$ Physicist, Advanced Systems Division, Mail Stop ASRC-15, AIAA Member Grade for first author.

${ }^{4}$ Chief Surface Systems Office, NE-S, KSC, and AIAA Member Grade for third author. 


\section{Introduction}

The LER provides a very diverse mobility platform, which allows it to interface with a variety of implements. Implements will provide the LER with the capabilities to perform tasks such as lunar regolith handling (to include dozing, grading, and excavation), equipment transport, and science operations. The LER provides a space for these implements to mount on the rear of the chassis. Current implements include a science tool kit and the light weight grader blade (Lance). These implements are manually attached and detached using the LER's original interface. This interface consists of five horizontal male pegs and two vertical sockets on the rear of the chassis.

Once an implement is mounted to the LER, clevis pins are inserted to lock the implement to the frame. This interface requires tight tolerances to reduce loads, which makes mate and demate operations very difficult. The Lance blade further complicates the problem do to its size and mass (4 meters long and $150 \mathrm{~kg}$ ). Furthermore, many of the proposed implements, such as the Lance Blade, are design to be tele-operated during a time that astronauts will not be present.

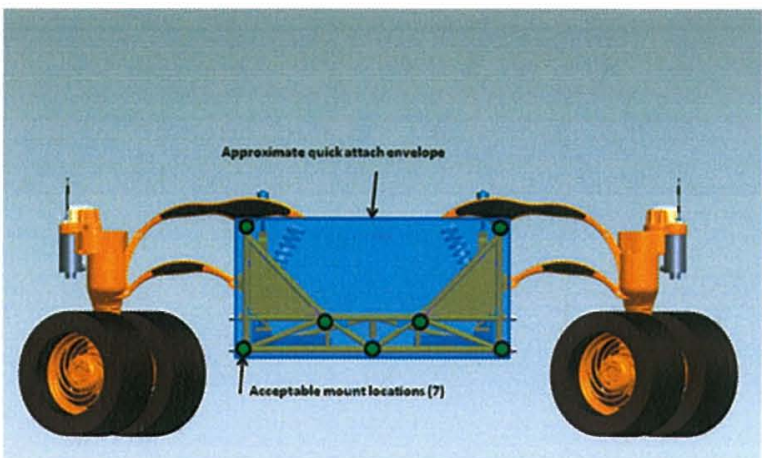
Operations like landing pad preparation and berm building, for example, are tasked to be completed before the astronauts land on the moon.

Based upon the limitations of the original mounting interface, requirements were generated for an automated quick attach mechanism. The general requirements are:

- The quick attach system shall mount to the LER via the 5 horizontal and 2 vertical sockets.

- The quick attach system shall provide one interface standard which implements must adopt to interface with the quick attach system.

- Mating tolerance:

○ Vertical ( $\left.\pm .5^{\prime \prime}\right)$, Horizontal $\left( \pm .5^{\prime \prime}\right)$, Roll $\left( \pm 5^{\circ}\right)$, Pitch $\left( \pm 5^{\circ}\right)$, Yaw $\left( \pm 5^{\circ}\right)$

- Quick Attach shall provide an umbilical connection, which will be independent from physical mate.

O Quick Attach umbilical shall provide a minimum of 12 (24 AWG) conductors for CAN and Ethernet protocols, a minimum of 2 (12 AWG) and 2 (4 AWG) conductors for power connections, and a minimum two fluid connectors.

- Instrumentation shall confirm the following states (in chronological order):

- First mate (Soft dock), Physically Latched (Hard dock), Umbilical Latched, Umbilical Unlatched, Physically Unlatched (No contact).

- While undocked, implements must rest on the surface within $\pm 10^{\circ}$ from a plane normal to the ground.

- The quick attach system shall incorporate an EVA assist. 


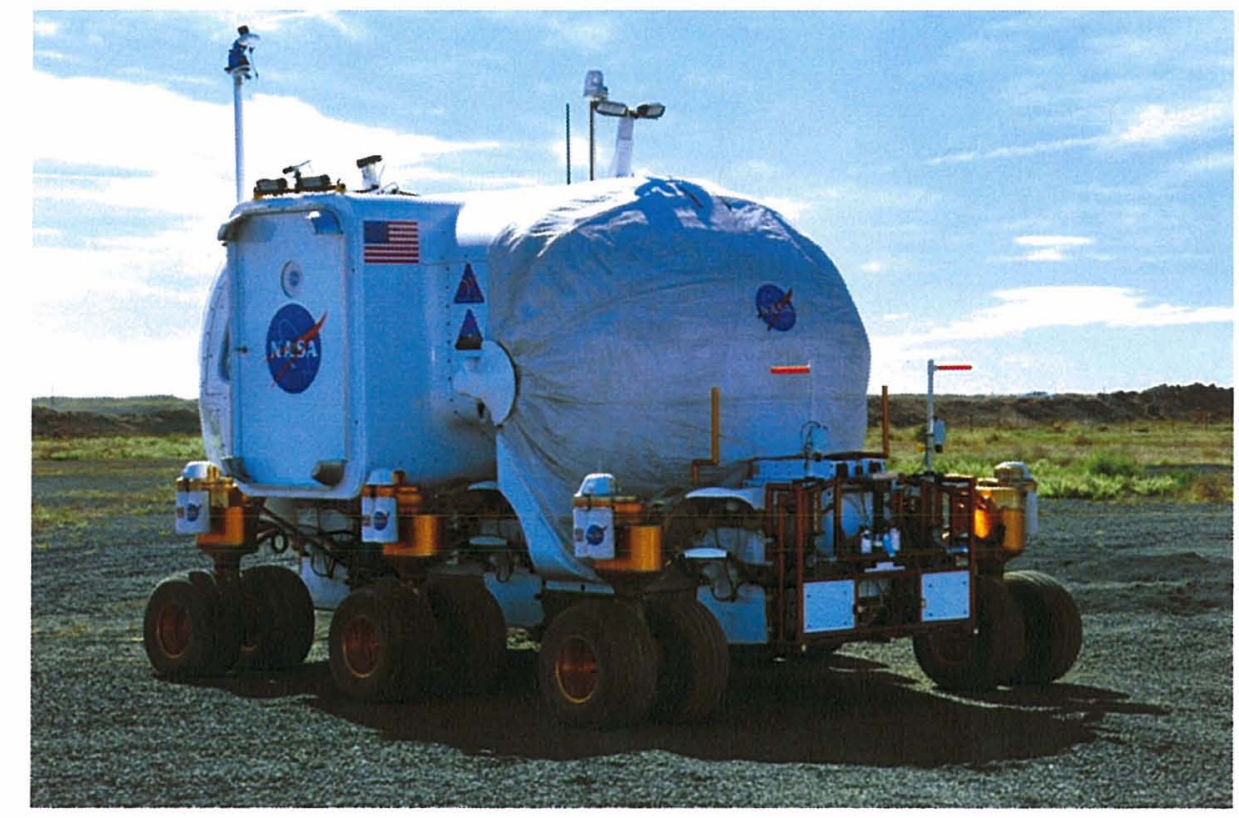

\section{System Overview}

The Quick Attach system consists of a structural frame that is mounted to the LER's original interface. This frame is the interconnect between the rover and the Quick Attach mechanism. The Quick Attach mechanism consists of an upper and lower engagement bar that captures 4 hooks mounted to an implement. The upper bar is connected to a ball screw giving it the capability to be actuated up and down. When the upper bar is in the lowest position the implement's hooks can fit between the engagement bars. Once the upper hooks are positioned over the upper bar, it is actuated to a position where upper hooks are fully engaged starting to lift the implement off the ground (Soft Dock). During this stage the implement is aligned to the QA using the upper hooks cam surface and the alignment cones on either end of the upper engagement bar. The LER's suspension is then raised to its nominal ride height. The upper bar is subsequently actuated until a preload value of $1360 \mathrm{~kg}$ is reach on the load cell (Hard Dock).

During the design selection of the Quick Attach numerous concepts were considered, the horizontal engagement bar design was chosen early on for its simplistic operation. With this horizontal design there were two concepts for implementing the actuation, linear and rotational actuation. Due the high moment load from lifting implements, in the rotational concept, the linear actuation was preferred. An over center mechanism was also considered for both the linear and rotational design, but the added risk during operation outweighed any advantages. Over center mechanisms are still being considered for future generations of the Quick Attach.
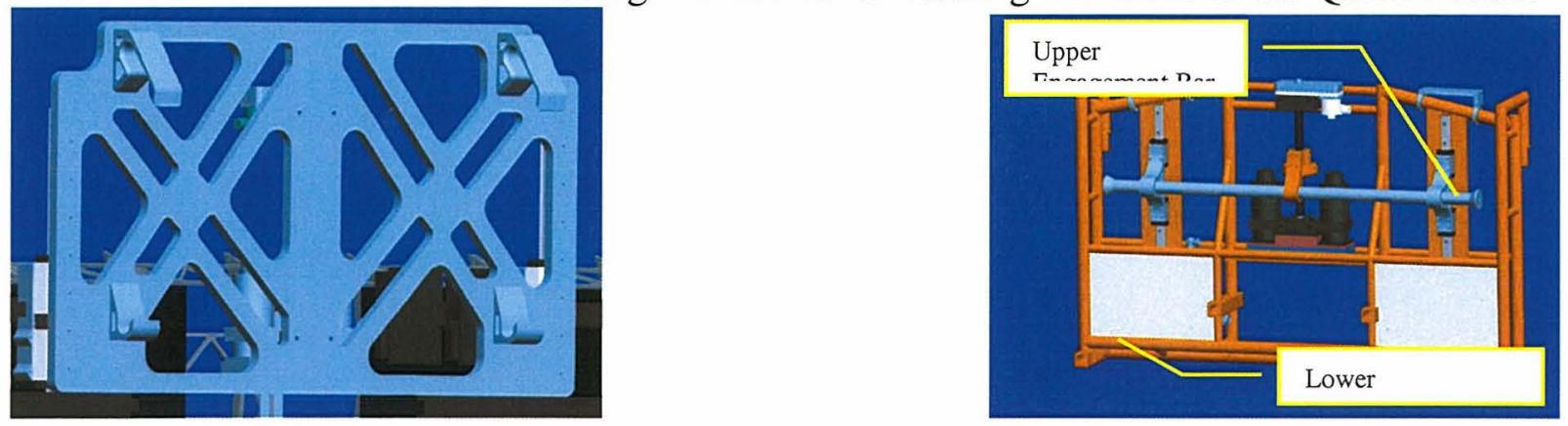

American Institute of Aeronautics and Astronautics 


\section{Mechanical Interface}

The structural frame of the Quick Attach system is a weldment of coped round 4130 chromoly tubes. An important design requirement was the need to keep the quick attach system built in as close to the vehicle as possible. At the start of the project the CAD models were approached with a top down design. Skeleton models were utilized within Pro/Engineer to allow the designers to easily change the dimensions of the structural frame as various components were identified and analyses were completed. The skeleton model approach also integrated well into the structural analysis using Pro/Mechanica

Skeleton models, machined tubes, weld clamps, weld wire used and reasoning, Faro data to get alignment single mill setup...

The linear slide assembly consists of two Nook Industries profile rails, parallelism using single mill setup...

The actuator assembly consists of the Nook ballscrew, load cell, gear box (dual input single out, torque limited), output rotational encoder, override mechanism...

The quick attach (QA) system is a retro fit to the Chariot vehicle (a.k.a. Lunar Electric Rover). The QA gives Chariot the ability to dock and undock with various implements on the fly. The QA consists of a welded 4130 Chrome-moly support frame, an actuation stage, and a control system. The support frame for the QA was designed to house the controls, actuation stage, and also act as one of the mating cam surfaces. The frame is made from coped 4130 round tubes and has mounting points that match up to the Chariot chassis. Of these mounting points, five are bored out sockets that slip over pins on Chariot and two are sockets that accept adjustable struts. The interface locations were critical to the design because of the large loads that the interface might see due to implements such as dozer blades and excavators. If the mounting tolerances were too loose the system could load unevenly and induce stress concentrations. In order to minimize this problem the team used a Faro portable CMM to take measurements of the various Chariot frames. The data were merged and averaged into a common drawing and the result was mounting locations that fit tightly on each of the frames. Attached to the frame is the actuation stage. The QA picks up implements by capturing two cam-like rods in-between a set of hooks on an implement. In order to do this a surface on the bottom of the support frame acts as the lower cam surface and the upper cam surface is a rod that is attached to linear slide rails and actuated vertically. When Chariot approaches an implement it puts the upper rod in its lowest position and drives in-between the hooks on the implement. At this point the upper rod is actuated to the top of its travel. As the rod moves up it engages the hooks and uses alignment cones and chamfers to self center the implement. After a certain distance the implement lifts off the ground completely and both upper and lower rods are engaged in hooks, at which point they are preloaded to ensure a positive lock. In order to complete this actuation two liner profile rails are placed outboard of a central ball screw. The rails and ball screw are coupled together with the upper rod. When the QA is aligned to pick up an implement, the hooks on the implement sit just outboard of the linear rails. This feature helps to keep the moments away from the central ball screw and prolong its lifetime. The ball screw is actuated through a novel dual motor input gearbox (NTR submitted) on one end, and is coupled to a absolute position encoder and an override $3 / 4$ " hex nut on the other. The control system consists of motor controllers, a NI C-Rio controller, network switch, and power transformers. The system controls the actuation of the QA and monitors the preload value from the load cell as well as the condition of the torque limiter, and communicates with the

4

American Institute of Aeronautics and Astronautics 
Chariot control system for integration into the cabin computers. This system has been outlined in more detail in two other NTRs (one hardware and one software).

\section{Control System}

The Quick Attach Control System was specifically designed to perform mate/demate operations of the Quick Attach mechanism. Figure 1 shows the overall schematic of the control system. The National Instruments Compact RIO platform was selected to be the central controller for the Quick Attach mechanism. The four slot version of this chassis (model 9101) is small enough to fit inside the frame hardware yet flexible enough to connect with the diverse set of pre-existing transducers and motor controllers. The central controller run VxWorks and is model number 9012.

The SICK Absolute encoder (Model ATM60-AAA12X12) is the primary means of transitioning between the various states of the system. The encoder broadcasts the SSI protocol to the JSC-REAM Board. The REAM board is polled by the cRIO system via the CAN network to obtain the absolute encoder position. Calibration of the stage will zero the system and knowing the lead of the screw $(0.25 \mathrm{inch} / \mathrm{rev})$ enables determination of absolute position relative to the zero position. At any instant, if the CAN communication is lost with the REAM board, the motion is stopped on the motors. The REAM board also controls the servo motor brakes.

The ball screw stage is equipped with a safety coupling that releases if the torque on the motor exceeds a threshold value. A Omron proximity sensor detects disengagement of the safety coupling and is read by the cRIO via a 24 volt digital input module (NI 9421). The sensor is configured as Normally Closed to ensure that it is connected. The proximity sensor goes low $(0$ $\mathrm{V})$ upon safety coupling detection. All motion is halted when the proximity sensor goes low.

The ball screw is actuated via dual (redundant) Magmotor servo motors (Model 730340023). The motors are controlled directly by Advanced Motion Control servo drives (Model dc201e30a40ldc). The torque on the motors are sufficient that the stage can be actuated with a single motor, in the event of a failure. The AMC drives are configured to operate in velocity mode. They are limited with a maximum drive current (well below their rated spec) to ensure safety of the mechanical systems. Additionally, "velocity following fault" detection is enable such that if the actual motor velocity does not reach and maintain the commanded velocity within certain timing parameters, drive motion is stopped. This is and additional measure to

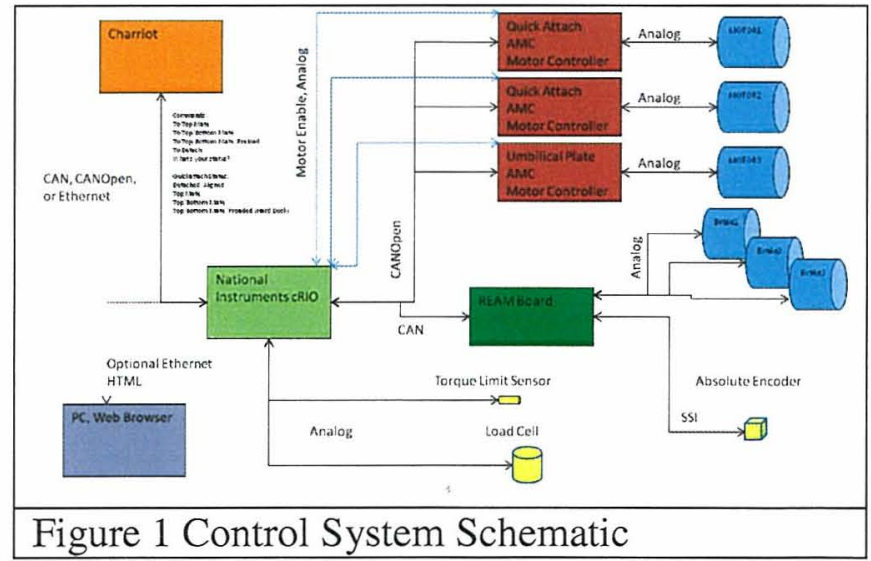
ensure that if the drive binds or hits a stop that the motors will stop driving.

The AMC servo drives are controlled by the cRIO via CAN module 9853. The CANOpen protocol over CAN is used to communicate to the AMC drives. If at any time the CAN connection drops from the $\mathrm{RIO}$ to the AMC modules, the voltage enable line on the AMC drives is brought low with the cRIO module 9472, disabling the motor drive. The preload on the ball screw stage is monitored with an Omega load cell (model 8313-213-5K). This sensor is a full wheatstone bridge configuration with a full scale range of $5000 \mathrm{lbf}$. The sensors is connected to a cRIO module 9219 which has a sensitivity of $7 \mathrm{mV} / \mathrm{V}$, resulting in an effective resolution of $0.002 \mathrm{lbf}$. 
The control system software consists of two parts: the FPGA software and the Real-Time OS software (VX Works). Both pieces of software are written in National Instruments LabVIEW, version 8.6.1. The FPGA software was written using the LabVIEW FPGA module. The bulk of the code on the FPGA module is National Instruments CAN engine for input/output to the CAN bus. This software allows various CAN software layers (CAN, CanOPEN, J1939, etc...) to use the same CAN bus simultaneously. Additionally, some parallel loops were added to provide an interface to the other hardware I/O: the 9119,9421 , and 9472 modules. Since high speed data transfer for these modules was not needed, the interface was through the FPGA front panel (as opposed to a RT FIFO or DMA).

The software running on the Real Time portion of the controller(VX Works)was written in LabVIEW RT. The main loop of the RT software is written as a Queued State Machine architecture. There are three other loops that affect the state machine: a user interface loop, a NI Shared Variable remote control access loop, and a TCP/IP Sockets remote control access loop. The RT software contains access to a subset of the CANOpen object dictionary of the AMC controllers and REAM board. The cRIO is intended to operate "headless",

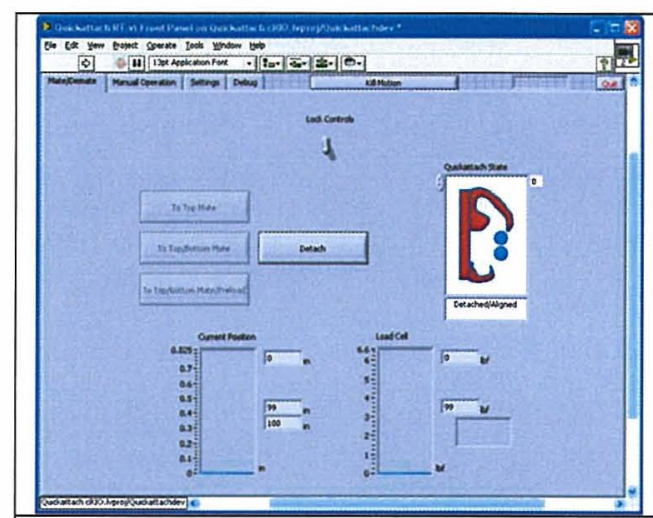

Figure 2 Main front panel display of software. however, the front panel of the software can be viewed with a web browser pointed at the hardware. Figure 2 shows a snapshot of the main display of the front panel. The Quick Attach mechanism can either be controlled from this front panel through a web browser or via any of the remote control mechanisms. The front panel interface allows full manual control over the drives and contains additional debugging information.

\section{Lunar Analog Testing}

The quick attach system was demonstrated at the Desert Research and Technology Studies (Desert RATS) lunar analog field test outside of Flagstaff, Arizona in September 2009. Two test subjects, Astronaut Michael Gernhardt and Geologist Brent Garry, lived in and operated the LER for 14 consecutive days. On the 11th day of the mission the quick attach was installed to the rear of LER with the intent of testing ease of use in terms of alignment cues, control panel interfaces, and time for mate/demate.

The quick attach was tested at Johnson Space Center during two dry runs in preparation for the Desert RATS field test. During these tests the team learned that macroscopic views and alignment cues provided the easiest and fastest approach when aligning to an implement. The first alignment iteration was a camera located on the quick attach with a reticle overlay on the display and a target attached to the implement. The performance of this type of targeting system was limited because the focus and zoom lengths on the camera available could not clearly resolve the target until LER was less than a meter away. The large adjustments at such a short distance from the implement were difficult for the LER which is more suited to a rolling approach with fine adjustments. In addition the target and reticle method understated the tolerance of the quick attach, giving users the impression they had to line up the cross-hairs perfectly to achieve the capture envelope. Alternatively a camera positioned on the top rear of the pressurized cabin provided an elevated central view from which the user could easily identify 
features on the quick attach and implements. In the second dry run, 3 foot vertical poles were attached to the quick attach and LANCE blade and were used as alignment cues that from the cabin camera provided visual yaw, roll, depth, and transverse positioning feedback. Additional camera views were provided on the quick attach poles giving a plan view over the left and right upper mating surfaces. With these features and views users were able to approach implements and make necessary adjustments while rolling toward them. Once the hooks of the implement came within view of the pole mounted cameras the user could slowly make any final adjustments until both hooks were within the capture envelope. This approach worked well and suggestions were made to include a feature that would provide feedback on the relative height between the quick attach and the implement at the Desert RATS test. To accomplish this a horizontal section of pipe was attached to each of the poles on the quick attach and implement. During Desert RATS these features were tested but due to the installation of the cabana on the rear of the LER after the second dry run the camera view was obstructed and exact placement of the alignment poles could only be estimated with the time available.

During the day 11 testing the quick attach was mounted to the rear of the LER and the LANCE blade placed in an open gravel yard. Because of an IP address conflict the integrated quick attach control panel was unavailable from the driver station. Fortunately a backup LabView control panel was available and allowed the same functionality as the integrated version. LER performed a series of mate and demate operations using only the feedback provided by the cameras and quick attach control system. The inexact positioning of the alignment poles (specifically the height feature) caused the alignment attempts to be repeatedly high. Once this was observed and relayed to the drivers the mate and demate operations lasted on average under 3 minutes. A human factors study was performed by the LER team in which numerical ratings (1-10, 1 being best) were given to a number of tasks and relative complexity. The quick attach system scored one of the highest ratings out of the 14 day mission with scores of 2 for control interfaces, and 2 for task complexity.

Following the testing during the primary mission the quick attach was installed on the Chariot Chassis B. Chassis B was a recently built rover at the time and was scheduled to be driven extensively during the Desert RATS tests to prove out the system. The quick attach piggybacked on this opportunity and was run through its paces. Chassis B was also without a pressurized cabin and therefore lacked cameras to use during QA operations. However, from a distance of over 50 meters the Chassis B rover was aligned and mated to the LANCE blade repeatedly in less than 1 minute. The method that accomplished this was driving the vehicle toward the implement at a nominal low height while adjusting for yaw in the approach until it reached the implement and slowly nudged it to confirm contact. The vehicle then lifted its chassis and if the implement was captured it would lift off the ground. If the implement was not captured it would not lift with the chassis, and the driver would then lower the vehicle past its previous setting and move forward into the capture envelope.

\section{Future Work}

At the DRATS field test the Quick Attach system was proven to be a valuable asset to the LER's operations. Due to this success future implements will be required to use the QA's interface. A interface document is available for any implement needing to interface with the LER. Currently the LER's science tool kit/fireman's platform, Roxygen $\mathrm{O}_{2}$ excavator, and 
Langley's Portable Utility palette are being fitted with hooks and is scheduled for testing with the Quick Attach during the 2010 DRATS field test.

The first prototype Quick Attach system included the physical mate operation, but design of an dust tolerant blind mate umbilical is currently in work. A location on the structural frame has been left open for the implementation of the umbilical. A proof of concept umbilical was tested this past year to help determine the appropriate sizing as well as design selection.

A second generation Quick Attach is also in work for the 2010 DRATS field test. This generation will not change the interface requirements from the previous design, but incorporate a cleaner implementation. A lighter weight version, possibly made from composites is also in work.

A second generation LER is being designed at JSC and it is planned to incorporate a QA design into the chassis. A KSC team is with working JSC to provide lessons learned and requirements for this design.

\section{Conclusion}

A conclusion section is not required, though it is preferred. Although a conclusion may review the main points of the paper, do not replicate the abstract as the conclusion. A conclusion might elaborate on the importance of the work or suggest applications and extensions. Note that the conclusion section is the last section of the paper that should be numbered. The appendix (if present), acknowledgment, and references should be listed without numbers.

\section{Acknowledgments}

This task was possible due to the financial and management support of the NASA Constellation, Exploration Technology Development Program (ETDP), and in particular the Human Robotics Systems (HRS) project. The authors would like to thank Dr. Robert Ambrose of the HRS project and Mr. Chris Culbert of the Lunar Surface Systems office.

\section{References}

The following pages are intended to provide examples of the different reference types, as used in the AIAA Style Guide. When using the Word version of this template to enter references, select the "references" style from the drop-down style menu to automatically format your references. If you are using a print or PDF version of this document, all references should be in 9-point font, with reference numbers inserted in superscript immediately before the corresponding reference. You are not required to indicate the type of reference; different types are shown here for illustrative purposes only.

\section{Periodicals}

${ }^{1}$ Vatistas, G. H., Lin, S., and Kwok, C. K., "Reverse Flow Radius in Vortex Chambers," AIAA Journal, Vol. 24, No. 11, 1986, pp. 1872, 1873.

${ }^{2}$ Dornheim, M. A., "Planetary Flight Surge Faces Budget Realities," Aviation Week and Space Technology, Vol. 145, No. 24, 9 Dec. 1996, pp. 44-46.

${ }^{3}$ Terster, W., "NASA Considers Switch to Delta 2," Space News, Vol. 8, No. 2, 13-19 Jan. 1997, pp., 1, 18.

All of the preceding information is required. The journal issue number ("No. 11" in Ref. 1) is preferred, but the month (Nov.) can be substituted if the issue number is not available. Use the complete date for daily and weekly publications. Transactions follow the same style as other journals; if punctuation is necessary, use a colon to separate the transactions title from the journal title. 
Books 14.

${ }^{4}$ Peyret, R., and Taylor, T. D., Computational Methods in Fluid Flow, $2^{\text {nd }}$ ed., Springer-Verlag, New York, 1983, Chaps. 7 ,

${ }^{5}$ Oates, G. C. (ed.), Aerothermodynamics of Gas Turbine and Rocket Propulsion, AIAA Education Series, AIAA, New York, 1984, pp. 19, 136.

${ }^{6}$ Volpe, R., "Techniques for Collision Prevention, Impact Stability, and Force Control by Space Manipulators," Teleoperation and Robotics in Space, edited by S. B. Skaar and C. F. Ruoff, Progress in Astronautics and Aeronautics, AIAA, Washington, DC, 1994, pp. 175-212.

Publisher, place, and date of publication are required for all books. No state or country is required for major cities: New York, London, Moscow, etc. A differentiation must always be made between Cambridge, MA, and Cambridge, England, UK. Note that series titles are in roman type.

Proceedings

${ }^{7}$ Thompson, C. M., "Spacecraft Thermal Control, Design, and Operation," AIAA Guidance, Navigation, and Control Conference, CP849, Vol. 1, AIAA, Washington, DC, 1989, pp. 103-115

${ }^{8}$ Chi, Y., (ed.), Fluid Mechanics Proceedings, SP-255, NASA, 1993.

'Morris, J. D. "Convective Heat Transfer in Radially Rotating Ducts," Proceedings of the Annual Heat Transfer Conference, edited by B. Corbell, Vol. 1, Inst. Of Mechanical Engineering, New York, 1992, pp. 227-234.

At a minimum, proceedings must have the same information as other book references: paper (chapter) and volume title, name and location of publisher, editor (if applicable), and pages or chapters cited. Do not include paper numbers in proceedings references, and delete the conference location so that it is not confused with the publisher's location (which is mandatory, except for government agencies). Frequently, CP or SP numbers (Conference Proceedings or Symposium Proceedings numbers) are also given. These elements are not necessary, but when provided, their places should be as shown in the preceding examples.

Reports, Theses, and Individual Papers

${ }^{10}$ Chapman, G. T., and Tobak, M., "Nonlinear Problems in Flight Dynamics,” NASA TM-85940, 1984.

${ }^{11}$ Steger, J. L., Jr., Nietubicz, C. J., and Heavey, J. E., "A General Curvilinear Grid Generation Program for Projectile Configurations," U.S. Army Ballistic Research Lab., Rept. ARBRL-MR03142, Aberdeen Proving Ground, MD, Oct. 1981.

${ }^{12}$ Tseng, K., "Nonlinear Green's Function Method for Transonic Potential Flow," Ph.D. Dissertation, Aeronautics and Astronautics Dept., Boston Univ., Cambridge, MA, 1983.

Government agency reports do not require locations. For reports such as NASA TM-85940, neither insert nor delete dashes; leave them as provided by the author. Place of publication should be given, although it is not mandatory, for military and company reports. Always include a city and state for universities. Papers need only the name of the sponsor; neither the sponsor's location nor the conference name and location are required. Do not confuse proceedings references with conference papers.

\section{Electronic Publications}

CD-ROM publications and regularly issued, dated electronic journals are permitted as references. Archived data sets also may be referenced as long as the material is openly accessible and the repository is committed to archiving the data indefinitely. References to electronic data available only from personal Web sites or commercial, academic, or government ones where there is no commitment to archiving the data are not permitted (see Private Communications and Web sites).

${ }^{13}$ Richard, J. C., and Fralick, G. C., "Use of Drag Probe in Supersonic Flow," AIAA Meeting Papers on Disc [CD-ROM], Vol. 1, No. 2, AIAA, Reston, VA, 1996.

${ }^{14}$ Atkins, C. P., and Scantelbury, J. D., "The Activity Coefficient of Sodium Chloride in a Simulated Pore Solution Environment," Journal of Corrosion Science and Engineering [online journal], Vol. 1, No. 1, Paper 2, URL: http://www.cp/umist.ac.uk/JCSE/vol1/vol1.html [cited 13 April 1998].

${ }^{15}$ Vickers, A., "10-110 mm/hr Hypodermic Gravity Design A," Rainfall Simulation Database [online database], URL: http://www.geog.le.ac.uk/bgrg/lab.htm [cited 15 March 1998].

Always include the citation date for online references. Break Web site addresses after punctuation, and do not hyphenate at line breaks.

Computer Software

${ }^{16}$ TAPP, Thermochemical and Physical Properties, Software Package, Ver. 1.0, E. S. Microware, Hamilton, OH, 1992.

Include a version number and the company name and location of software packages.

Patents 
Patents appear infrequently. Be sure to include the patent number and date.

${ }^{17}$ Scherrer, R., Overholster, D., and Watson, K., Lockheed Corp., Burbank, CA, U.S. Patent Application for a "Vehicle," Docket No. P-01-1532, filed 11 Feb. 1979.

\section{Private Communications and Web Sites}

References to private communications and personal Web site addresses are generally not permitted. Private communications can be defined as privately held unpublished letters or notes or conversations between an author and one or more individuals. They may be cited as references in some case studies, but only with permission of the AIAA staff. Depending on the circumstances, private communications and Web site addresses may be incorporated into the main text of a manuscript or may appear in footnotes.

\section{Unpublished Papers and Books}

Unpublished works can be used as references as long as they are being considered for publication or can be located by the reader (such as papers that are part of an archival collection). If a journal paper or a book is being considered for publication choose the format that reflects the status of the work (depending upon whether it has been accepted for publication):

${ }^{18}$ Doe, J., "Title of Paper," Name of Journal (to be published).

${ }^{19}$ Doe, J., "Title of Chapter," Name of Book, edited by... Publisher's name and location (to be published).

${ }^{20}$ Doe, J., "Title of Work," Name of Archive, Univ. (or organization) Name, City, State, Year (unpublished).

Unpublished works in an archive must include the name of the archive and the name and location of the university or other organization where the archive is held. Also include any cataloging information that may be provided. Always query for an update if a work is about to be published. 